\title{
Acute Kidney Infarction Due to Left Ventricular Thrombus Embolization In Patient with Isolated Left Ventricular Noncompaction: A Case Report
}

\author{
Liqian Xu, Ji Yang, Yunmei Yang \\ Department of Geriatrics, The First Affiliated Hospital, School of Medicine, Zhejiang University, Hangzhou, China
}

\section{ABSTRACT}

Noncompaction of the ventricular myocardium (NVM) is a rare congenital cardiomyopathy that is characterized by multiple prominent trabeculations and deep intertrabecular recesses, and occurs due to arrest of normal embryogenesis of the endocardium and myocardium. It is also referred to as isolated left ventricular noncompaction (LVNC), because lesions are mainly in the left ventricle. The main clinical manifestations are heart failure, arrhythmia, systemic embolism, and sudden death. Systemic embolism is related to the occurrence of atrial arrhythmias or thrombus formation in the left ventricle. Most resulting thromboembolisms are cerebral or in the arteries of the lower limbs, and renal artery embolisms are rare. There are reports of a few previous cases of renal infarction with diagnoses of NVM on echocardiography, but a thrombus from the left ventricle has never been identified as the cause of a renal artery embolism. This paper reports a 53-year-old male who was admitted to our hospital for LVNC and renal infarction. He had a history of atrial fibrillation 3 years previously. We diagnosed LVNC with a thrombus in the left ventricle using echocardiography. The patient was discharged after renal replacement therapy and treatment with an anticoagulant. During the 2 years of follow-up, his condition remained stable.

\section{INTRODUCTION}

Noncompaction of the ventricular myocardium (NVM), also known as isolated left ventricular noncompaction (LVNC), is a rare congenital disease that can exist independently, but is also associated with other congenital cardiac anomalies and genetic disorders [Pantazis 2009; Bione 1996]. Engberding and Bender first described LVNC in 1984 [Engberding 1984], and the American Heart Association classified LVNC as a primary genetic cardiomyopathy in 2006 [Maron 2006]. In contrast, the European Society of Cardiology characterized LVNC as an "un-classified cardiomyopathy" in 2008 [Elliott 2008]. There are numerous reports of familial and sporadic cases [Stöllberger 2004; Ozkutlu 2002; Ritter 1997]. Its clinical manifestations include heart failure,

Received May 19, 2016; received in revised form fanuary 4, 2017; accepted fanuary 30, 2017.

Correspondence: Yunmei Yang, Department of Geriatrics, The First Affiliated Hospital, School of Medicine, Zhejiang University, Hangzhou, China; (e-mail:yyzkyq@163.com). arrhythmia, systemic embolism, and sudden death [Oechslin 2000; Pignatelli 2003]. The systemic embolism is related to the occurrence of atrial arrhythmias or thrombus formation in the left ventricle.

The most common thromboembolic events are cerebral and in the arteries of the lower limbs. There have been reports of renal artery embolisms, but no reports of a renal artery embolism caused by a left ventricular thrombus. Here, we present a 53-year-old male who was diagnosed with LVNC and renal infarction due to a left ventricular thromboembolism.

\section{CASE REPORT}

A 53-year-old male Han Chinese worker was admitted to our emergency department following onset of a sudden left-lower abdominal pain and nausea in January 2014. At admission, his blood pressure was $129 / 86 \mathrm{mmHg}$ and his heart rate was 105 beats $/ \mathrm{min}$. A physical examination indicated tenderness in the left abdomen. His laboratory data showed an elevated white blood cell count $(19.8 \times$ $10^{9} / \mathrm{L}$, with $86.6 \%$ neutrophils), high blood urea nitrogen $(14.5 \mathrm{mmol} / \mathrm{L})$, high serum creatinine $(4.95 \mathrm{mg} / \mathrm{dL})$, and elevated B-type natriuretic peptide $(1791.6 \mathrm{pg} / \mathrm{mL})$. Blood electrolyte levels were normal. Arterial blood-gas sampling indicated metabolic acidosis $(\mathrm{pH}:$ 7.32, base excess: $-6.0 \mathrm{mEq} / \mathrm{L}$ ). His chest radiography and other laboratory data, including amylase and cardiac enzyme levels, were unremarkable. Three years prior to presentation, he presented with exertional dyspnea, and was diagnosed with dilated cardiomyopathy and atrial fibrillation. Coronary angiography showed that his left ventricle was larger, and most of the blood supply to the myocardium was from the right coronary artery. There was no obvious stenosis in the trunk of the left or right coronary arteries. There were different degrees of stenosis in the proximal segment of the left anterior descending artery (LAD), the distal segment of left circumflex artery (LCX), and the distal region of the right coronary artery (RCA), but stenosis in each was less than $30 \%$. He reported long-term oral intake of a beta-blocker, diuretics, an angiotensin converting enzyme inhibitor (ACEI), digoxin, and aspirin. However, after 9 months, he discontinued aspirin because of discomfort in the upper abdomen. He reported smoking 20-40 cigarettes per day for 30 years, and occasionally drinking alcohol. He had none of the classic cardiovascular risk factors, such as diabetes or hypertension, and no family history of cardiomyopathy or heart failure. An 

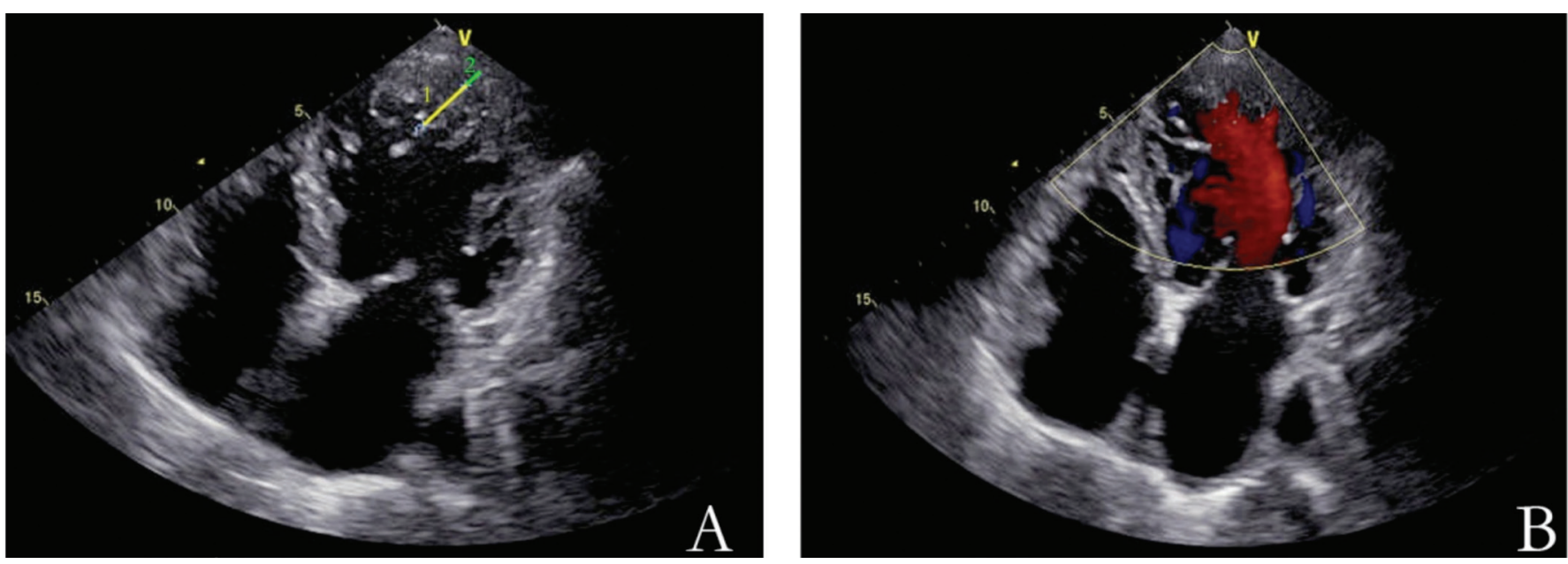

Figure 1. Transthoracic echocardiography. (A) Echocardiographic 4-chamber view showing the ratio of noncompacted (yellow) to compacted (green) myocardium was more than 2:1, 1-1.84 cm and 2-0.52 cm as measured. (B) Color Doppler image showing blood flow (red) in the recess between trabeculae.

electrocardiogram at admission indicated atrial fibrillation, nospecificST changes, and a ventricular rate of 105 beats $/ \mathrm{min}$. We performed an abdominal ultrasonogram because of his abdominal pain. Two wedge-shaped areas which had lost blood supply were detected in the upper pole of the left kidney, and right kidney atrophy, although the patient denied any history of chronic nephropathy. His kidney function was normal, and he reported no abdominal pain or hematuria before presentation. We speculated that his atrophic right kidney was congenital. The abdominal ultrasonogram indicated left renal infarction. To identify the source of the embolism, we performed transthoracic echocardiography. The results indicated the patient had an enlarged left atrium (LA) diameter: $48 \mathrm{~mm}$ ) and left ventricle (LV) (diameter at diastole: $60 \mathrm{~mm}$, diameter at systole: $52 \mathrm{~mm}$ ) and severely reduced LV function, with an estimated ejection fraction (EF) of $25 \%$. He also had mild mitral regurgitation and aortic regurgitation, diffuse weakness of the left ventricle wall motion, and a normal right ventricle with estimated systolic pressure of $53 \mathrm{mmHg}$, indicating pulmonary hypertension. In addition, there were prominent left ventricular trabeculations, and deep intertrabecular recesses communicating with the $L V$ cavity (Figure 1) that suggested the presence of noncompacted heart muscles. Moreover, there were high echo nodules (about $1.2 \times 1.2$ $\mathrm{cm}$ ) in the lateral wall of the LV (Figure 2), and fluttering during the cardiac contraction-diastolic period. We classified this as a left ventricular mural thrombus, and therefore diagnosed him with a left renal infarction and a left ventricle thrombus caused by LVNC. The day after admission, the patient presented with oliguria and hyperkalemia. He was given continuous renal replacement therapy (CRRT) because of multiple organ failure (MOF). After 1 week, he was given maintenance hemodialysis, 3 times per week. Low molecular weight heparin was given during the CRRT and hemodialysis. His kidney function returned to normal after 3 months. At that time, he was given warfarin, and his follow-up has been uneventful over the past 2 years.

\section{DISCUSSION}

In the early embryonic period, the human myocardium is a loose meshwork of interwoven myocardial fibers that enlarge the myocardial surface. As heart blood dispersion increases exchange, this provides nutrition for the heart. The fetal myocardium condenses after 8 weeks of gestation, following the formation of coronary blood vessels. This process typically progresses from the epicardium to the endocardium, and from the base of the heart to the apex, the same direction in which the coronary artery forms [Rooms 2015; Petersen 2005]. LVNC is a congenital disorder characterized by an arrested compaction of the loose meshwork of interwoven myocardial fibers during embryogenesis, and leads to multiple prominent trabeculations and deep intertrabecular recesses. The severity of the myocardial noncompaction depends on the time of its arrest [Jenni 1986; Bartram 2007]. Lesions mainly occur in the left ventricle, and there may or may not be right ventricular involvement. This abnormality may be isolated, or occur in association with a complex congenital heart disease or genetic disease.

The pathogenesis of LVNC is uncertain. It is a genetically heterogeneous disorder, and its occurrence clusters within families. Bione et al [Bione 1996] and Bleyl et al [Bleyl 1997] confirmed that mutations in the $\mathrm{Xq} 28$ gene $\mathrm{G} 4.5$ are related to $\mathrm{X}$-linked cardiomyopathy based on their studies of Barth syndrome. Ichida et al [Ichida 2001] found a missense mutation on chromosome $18 \mathrm{q} 12$, based on study of a Japanese family in which 5 members had LVNC with congenital heart defects, and 1 member had isolated LVNC. Other studies identified patients with LVNC who had mutations in genes encoding mitochondrial and sarcomere proteins [Probst 2011; Klaassen 2008; Tang 2010].

The diagnosis of LVNC relies mainly on echocardiography and cardiac magnetic resonance (CMR) imaging. Echocardiography is the most commonly used tool because it is simple and quick. The following echocardiographic diagnostic criteria recommended by Jenni et al are now widely 


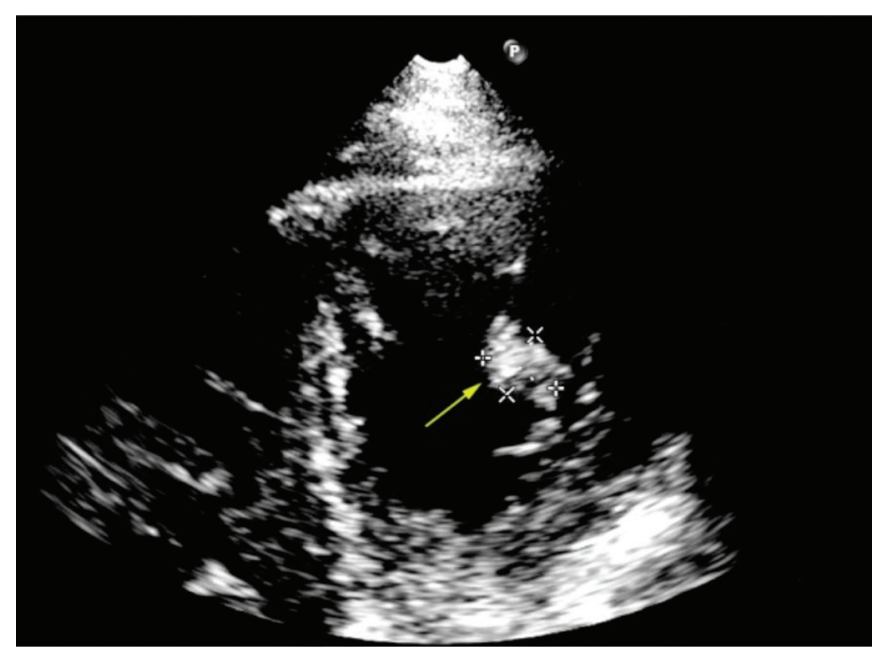

Figure 2. Transthoracic echocardiography demonstrating high echo nodules (about $1.2 \times 1.2 \mathrm{~cm}$ ) in the lateral wall of the LV (arrow).

accepted [Jenni 2001]: (a) N:C ratio greater than 2:1 at endsystole (N: noncompacted endocardial layer; C: compacted epicardial layer); (b) lesions of the myocardium mainly in the mid-lateral, mid-inferior, and apical areas; (c) deep intertrabecular recesses filled with blood from the LV cavity based on color Doppler ultrasonography; and (d) no evidence of other cardiac anomalies (for isolated LVNC). Our patient's echocardiogram showed an $\mathrm{N}: \mathrm{C}$ ratio of more than 2:1, and the color Doppler ultrasonography indicated flow in the recesses, thus confirming a diagnosis of LVNC. CMR imaging is increasingly used for the diagnosis of LVNC, as it provides clear images of cardiac morphology at high spatial resolution. CMR imaging is particularly useful for patients in whom the diagnosis is uncertain by echocardiography, although there are no accepted standards for using CMR imaging to diagnose LVNC. An N:C ratio greater than 2.3 measured at enddiastole is used as a diagnostic indicator for noncompaction cardiomyopathy [Petersen 2005].

Although LVNC is a congenital abnormality, the onset of symptoms varies widely, and most patients have no symptoms when they are young. Clinically, most patients present at middle-age when they begin to suffer from progressive cardiac dysfunction, systemic circulation embolism, and arrhythmia.

There are no specific treatments for the LVNC, and the main goals are management of symptoms and prevention of complications. The principles used to treat cardiac insufficiency are the same as those for dilated cardiomyopathy. There is still debate about the use of anticoagulation therapy for LVNC. Based on the high rate of embolic events reported in long-term follow-up data from 2000, Oechslin et al [Oechslin 2000] suggested a more aggressive approach to prescription of anticoagulants, independent of ventricular systolic function. However, in 2011 Oechslin and Jenni [Oechslin 2011] recommended anticoagulation therapy in patients with impaired systolic function (LV ejection fraction $<40 \%$ ), because deep intertrabecular recesses and slow blood flow might increase the risk of thrombus formation.
In the present case, the patient had 2 risk factors for systemic embolism: atrial fibrillation and left ventricular thrombus. We believe that the left ventricular thrombus in our patient was the source of the renal infarction because transthoracic echocardiography indicated no emboli in the left atrium. Pearson et al recommended use of transesophageal echocardiography to identify potential cardiac sources of emboli in most patients, even those with normal transthoracic echocardiograms [Pearson 1991]. In the present case, the presence of MOF after admission did not allow transesophageal echocardiography. Unfortunately, the patient refused transesophageal echocardiography as he improved. Anticoagulation therapy might still be needed in a patient with LVNC and a coexisting $\mathrm{LV}$ thrombus, even in the absence of systolic dysfunction or atrial fibrillation.

Different follow-up studies have reported different prognoses for LVNC patients. Ritter et al [Ritter 1997] reported that $47 \%(8 / 17)$ of patients with isolated LVNC died and $12 \%$ (2/17) received heart transplantations during a 6-year followup period. Oechslin et al [Oechslin 2000] reported that $35 \%$ (12/43) of patients with isolated LVNC died and 12\% (4/43) received heart transplantation during a $40 \pm 40$ month follow-up study. However, Murphy et al [Murphy 2005] studied 45 LVNC patients and reported better prognoses than the above two studies. In particular, they reported sudden cardiac death in a single 28-year-old patient with NYHA class III heart failure awaiting cardiac transplant, but the other 44 patients survived with or without transplantation during the 46-month follow-up. The conclusions of these studies differ because of differences in the study populations. Our follow-up of the patient in the present study indicated he remained stable after 2 years, and we plan to continue regular follow-ups.

\section{REFERENCES}

Bartram U, Bauer J, Schranz D. 2007. Primary noncompaction of the ventricular myocardium from the morphogenetic standpoint. Pediatr Cardiol 28:325-32.

Bione S, D'adamo P, Maestrini E, et al. 1996. A novel X-linked gene, G4.5. is responsible for Barth syndrome. Nat Genet 12:385-9.

Bleyl SB, Mumford BR, Brown-Harrison MC, et al. 1997. Xq28-linked noncompaction of the left ventricular myocardium: prenatal diagnosis and pathologic analysis of affected individuals. Am J Med Genet 72:257-65.

Elliott P, Andersson B, Arbustini E, et al. 2008. Classification of the cardiomyopathies: a position statement from the European Society of Cardiology Working Group on Myocardial and Pericardial Diseases. Eur Heart J 29:270-6.

Engberding R, Bender F. 1984. Identification of a rare congenital anomaly of the myocardium by two-dimensional echocardiography: persistence of isolated myocardial sinusoids. Am J Cardiol 53:1733-4.

Ichida F, Tsubata S, Bowles KR, et al. 2001. Novel gene mutations in patients with left ventricular noncompaction or Barth syndrome. Circulation 103:1256-63.

Jenni R, Goebel N, Tartini R, et al. 1986. Persisting myocardial sinusoids of both ventricles as an isolated anomaly: echocardiographic, 
angiographic, and pathologic anatomical findings. Cardiovasc Intervent Radiol 9:127-31.

Jenni R, Oechslin E, Schneider J, et al. 2001. Echocardiographic and pathoanatomical characteristics of isolated left ventricular non-compaction: a step towards classification as a distinct cardiomyopathy. Heart 86:666-71.

Klaassen S, Probst S, Oechslin E, et al. 2008. Mutations in sarcomere protein genes in left ventricular noncompaction. Circulation 117:2893-901.

Maron BJ, Towbin JA. 2006. Contemporary definitions and classification of the cardiomyopathies: an American Heart Association Scientific Statement from the Council on Clinical Cardiology, Heart Failure and Transplantation Committee; Quality of Care and Outcomes Research and Functional Genomics and Translational Biology Interdisciplinary Working Groups; and Council on Epidemiology and Prevention. Circulation 113:1807-16.

Murphy RT, Thaman R, Blanes JG, et al. 2005. Natural history and familial characteristics of isolated left ventricular non-compaction. Eur Heart J 26:187-92.

Oechslin EN, Attenhofer Jost CH, Rojas JR, et al. 2000. Long-term follow-up of 34 adults with isolated left ventricular noncom $\neg$ paction: a distinct cardiomyopathy with poor prognosis. J Am Coll Cardiol $36: 493-500$

Oechslin E, Jenni R. 2011. Left ventricular non-compaction revisited: a distinct phenotype with genetic heterogeneity? Eur Heart J 32:1446-56.

Ozkutlu S, Ayabakan C, Celiker A, et al. 2002. Noncompaction of ventricular myocardium: a study of twelve patients. J Am Soc Echocardiogr $15: 1523-8$.
Pantazis AA, Elliott PM. 2009. Left ventricular noncompaction. Curr Opin Cardiol 24:209-13.

Pearson AC, Labovitz AJ, Tatineni S, et al. 1991. Superiority of transesophageal echocardiography in detecting cardiac sources of embolism in patients with cerebral ischemia of uncertain etiology. J Amer Col Cardiol 17:66-72.

Petersen SE, Selvanayagam JB, Wiesmann F, et al. 2005. Left ventricular non-compaction: insights from cardiovascular magnetic resonance imaging. J Am Coll Cardiol 46:101-5.

Pignatelli R, McMahon JC, Dreyer WJ, et al. 2003. Clinical characterization of left ventricular noncompaction in children a relatively common form of cardiomyopathy. Circulation 108:2672-8.

Probst S, Oechslin E, Schuler P, et al. 2011. Sarcomere gene mutations in isolated left ventricular noncompaction cardiomyopathy do not predict clinical phenotype. Circ Cardiovasc Genet 4:367-74.

Ritter M, Oechslin E, Sütsch G, et al. 1997. Isolated noncompaction of the myocardium in adults. Mayo Clin Proc 72:26-31.

Rooms I, Dujardin K, De Sutter J. 2015. Non-compaction cardiomyopathy: a genetically and clinically heterogeneous disorder. Acta Cardiol 70:625-31.

Stöllberger C, Finsterer J. 2004. Left ventricular hypertrabeculation/ noncompaction. J Am Soc Echocardiogr 17:91-100.

Tang S, Batra A, Zhang Y, et al. 2010. Left ventricular noncompaction is associated with mutations in the mitochondrial genome. Mitochondrion 10:350-7. 